\title{
Non-perturbative aspects of gauge/gravity duality
}

\author{
Marco Billó, Marialuisa Frau, Luca Giacone* \\ Università di Torino, Dipartimento di Fisica and I.N.F.N. - Sezione di Torino \\ Via P- Giuria 1, I-10125 Torino, Italy \\ E-mail: billo, frau, giaconedto.infn.it
}

\author{
Alberto Lerda \\ Università del Piemonte Orientale, Dipartimento di Scienze e Innovazione Tecnologica \\ and I.N.F.N. - Gruppo Collegato di Alessandria - Sezione di Torino \\ Viale T. Michel 11, I-15121 Alessandria, Italy \\ E-mail: lerda@to.infn.it
}

\begin{abstract}
Recently we provided a microscopic derivation of the exact supergravity profile for the twisted scalar field emitted by systems of fractional D3-branes at a $\mathbb{Z}_{2}$ orbifold singularity. In this contribution we focus on a set-up supporting an $\mathscr{N}=2 \mathrm{SYM}$ theory with $\mathrm{SU}(2)$ gauge group and $N_{f}=4$. We take into account the tower of D-instanton corrections to the source terms for the twisted scalar and find that the profile of the latter can be expressed in terms of the chiral ring elements of the gauge theory. We show how the twisted scalar, which at the perturbative level represents the gravity counterpart of the gauge coupling, at the non-perturbative level is related to the effective gauge coupling in an interestingly modified way.
\end{abstract}

Proceedings of the Corfu Summer Institute 2012 "School and Workshops on Elementary Particle Physics and Gravity"

September 8-27, 2012

Corfu, Greece

\footnotetext{
*Speaker.
} 


\section{Introduction}

The embedding of supersymmetric gauge theories in a string framework using systems of D-branes has been very fruitful and inspiring for many developments. For example, the famous AdS/CFT correspondence [1] is rooted in the realization of the $\mathscr{N}=4$ super Yang-Mills (SYM) theory by means of D3-branes in flat space and in the profile of the supergravity bulk fields they induce in space-time. In less supersymmetric and/or in non-conformal cases (like the $\mathscr{N}=2$ gauge theories in four dimensions we will be interested in) the corresponding gravitational profile depends on some transverse directions representing the energy scale thus accounting for the running of the gauge theory. This fact was explicitly checked long ago [2] -[7] at the perturbative level in $\mathscr{N}=2$ SYM theories realized by fractional D3 branes of type IIB at non-isolated singularities, like for instance the $\mathbb{C}^{2} / \mathbb{Z}_{2} \times \mathbb{C}$ orbifold. By studying the emission of closed string fields from such branes, the corresponding "perturbative" supergravity solutions were constructed and it was found that a scalar field from the twisted sector, which we will call $t$, varies logarithmically in the internal complex direction $z$ transverse to the orbifold, matching precisely the perturbative logarithmic running of the gauge coupling with the energy scale. However, such perturbative solutions suffer from singularities at small values of $z$, i.e. in the IR region of the gauge theory, and have to be modified by non-perturbative corrections.

It is well-known that in $\mathscr{N}=2$ gauge theories there is a whole series of non-perturbative contributions to the low-energy effective action that are due to instantons. In the last two decades tremendous advances have been made in the study of instanton effects within field theory (for reviews, see for instance $[8,9]$ ), and more recently also within string theory by means of Dinstantons, i.e. D-branes with Dirichlet boundary conditions in all directions [10]-[13]. In the seminal papers $[14,15]$ the exact solutions for the low-energy effective $\mathscr{N}=2$ theories in the Coulomb branch, including all instanton corrections, were found using symmetry and duality arguments. In particular it was shown that the effective SYM dynamics in the limit of low energy and momenta can be exactly encoded in the so-called Seiberg-Witten (SW) curve which describes the geometry of the moduli space of the SYM vacua. Later these results were rederived from a microscopic point of view with the help of localization techniques $[16,17]$ that permit an explicit evaluation of the integrals over the multi-instanton moduli space. These techniques fit naturally in the string/D-brane context and indeed have been exploited for interesting generalizations of the SW results in many different directions.

It is then natural to ask how the infinite tower of instanton effects is encoded in the dual holographic description of the gauge theory in terms of gravity. To answer this question one possibility is to exploit symmetry and duality arguments and determine the background geometry that incorporates the exact SW solution, like in the M-theory constructions based on configurations of D4 and NS5 branes [18]. Another possibility is to compute directly the multi-instanton corrections to the profiles of the gravitational bulk fields. This is what we will discuss in this contribution, which heavily relies on the content of $[19,20,21]$ and especially of [22]. In particular we will briefly review how to derive the exact supergravity profile of the twisted field $t$ emitted by a system of fractional D3-branes at a $\mathbb{Z}_{2}$-orbifold singularity supporting a $\mathscr{N}=2$ quiver gauge theory with unitary groups and bi-fundamental matter, and show how to obtain from it the exact running of the gauge coupling constant, including the non-perturbative contributions, in perfect agreement with 


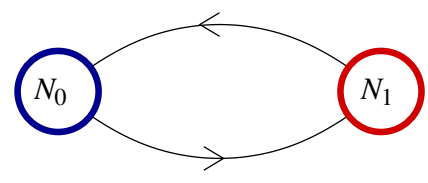

Figure 1: The quiver diagram for the orbifold $\mathbb{C}^{2} / \mathbb{Z}_{2}$. The branes of type 0 are represented by the blue circle while the branes of type 1 are represented by the red circle. The oriented lines connecting the two types of branes represent the hypermultiplets in the bifundamental representations.

the SW solution.

\section{The gauge coupling for the $\mathbf{S U}(2) N_{f}=4$ SYM theory}

We study the prototypical case of $\mathscr{N}=2$ SYM theories that are realized with fractional D3branes at the non-isolated orbifold singularity $\mathbb{C}^{2} / \mathbb{Z}_{2} \times \mathbb{C}$. In this orbifold there are two types of fractional D3-branes, which we call types 0 and 1, corresponding to the two different irreducible representations of $\mathbb{Z}_{2}$. The most general brane configuration therefore consists of $N_{0}$ branes of type 0 and $N_{1}$ branes of type 1 , and corresponds to an $\mathscr{N}=2$ quiver theory in four dimensions with gauge group $\mathrm{U}\left(N_{0}\right) \times \mathrm{U}\left(N_{1}\right)$ and with a matter content given by one hypermultiplet in the bifundamental representation $\left(\overline{\mathbf{N}}_{\mathbf{0}}, \mathbf{N}_{\mathbf{1}}\right)$ and one hypermultiplet in the $\left(\mathbf{N}_{\mathbf{0}}, \overline{\mathbf{N}}_{\mathbf{1}}\right)$ representation. The corresponding quiver diagram is represented in Fig. 1. Ignoring the gauge degrees of freedom on the $N_{1}$ branes, one obtains an $\mathscr{N}=2 \mathrm{U}\left(N_{0}\right)$ SYM theory with $2 N_{1}$ fundamental flavors and $\mathrm{U}\left(N_{1}\right)$ as global symmetry group. Furthermore, we will decouple the $\mathrm{U}(1)$ factors and concentrate on the $\mathrm{SU}\left(N_{0}\right) \times \mathrm{SU}\left(N_{1}\right)$ part of the symmetry group.

In this contribution we focus on the case $N_{0}=N_{1}=2$, representing an $\mathscr{N}=2 \mathrm{SU}(2) \mathrm{SYM}$ theory with $N_{f}=4$ flavors, but our results and methods apply to the general case as well [22]. The SU(2) $N_{f}=4 \mathrm{SYM}$ theory has a vanishing $\beta$-function but, when the flavors are massive, the gauge coupling gets renormalized at 1-loop by terms proportional to the mass parameters of the hypermultiplets. This situation corresponds to placing the fractional D3-branes at different positions in the transverse plane, i.e. to giving non-vanishing vacuum expectation values to the adjoint scalars $\phi_{0}$ and $\phi_{1}$ of the vector multiplets on the two types of branes according to

$$
\left\langle\phi_{0}\right\rangle=\operatorname{diag}(a,-a) \text { and }\left\langle\phi_{1}\right\rangle=\operatorname{diag}(m,-m) .
$$

Note that this brane configuration implies that the masses of the four flavors are given by

$$
\left\{m_{1}, m_{2}, m_{3}, m_{4}\right\}=\{m,-m, m,-m\} .
$$

In this case one finds that the perturbative part of gauge coupling constant

$$
\tau \equiv \frac{\theta_{\mathrm{YM}}}{\pi}+\mathrm{i} \frac{8 \pi}{g_{\mathrm{YM}}^{2}}
$$

is given by

$$
\mathrm{i} \pi \tau_{\text {pert }}=\mathrm{i} \pi t_{0}+\mathrm{i} \pi-\log 16+2 \log \left(1-\frac{m^{2}}{a^{2}}\right)
$$


where $t_{0}$ is the bare coupling. Besides these perturbative terms, there are also non-perturbative corrections due to instantons which can be explicitly computed using localization techniques (see for instance $[22,23]$ for details). The first two instanton contributions turn out to be given by

$$
\mathrm{i} \pi \tau_{\text {inst }}=q_{0}\left(\frac{1}{2}+\frac{3 m^{4}}{2 a^{4}}\right)+q_{0}^{2}\left(\frac{13}{64}+\frac{33 m^{4}}{32 a^{4}}-\frac{15 m^{6}}{8 a^{6}}+\frac{105 m^{8}}{64 a^{8}}\right)+\mathscr{O}\left(q_{0}^{3}\right)
$$

where $q_{0}=\mathrm{e}^{\pi \mathrm{i} t_{0}}$ is the instanton counting parameter. The complete effective coupling is therefore the sum of (2.4) and (2.5). For our future considerations it is convenient to rewrite it in terms of the gauge invariant quantity

$$
\mathbf{v}=\frac{1}{2}\left\langle\operatorname{tr} \phi_{0}^{2}\right\rangle
$$

which parametrizes the moduli space of the effective theory at the quantum level. Using the multiinstanton calculus and localization techniques, one can show that $\mathbf{v}$ is related to the classical vacuum expectation value $a$ in the following way [24,22]

$$
\mathbf{v}=a^{2}\left(1+q_{0} \frac{\left(a^{2}-m^{2}\right)^{2}}{2 a^{4}}+q_{0}^{2} \frac{\left(a^{2}-m^{2}\right)\left(13 a^{6}-15 a^{4} m^{2}+7 a^{2} m^{4}-5 m^{6}\right)}{32 a^{8}}+\mathscr{O}\left(q_{0}^{3}\right)\right)
$$

Inverting this relation and substituting it into (2.4) and (2.5), after some simple algebra we find

$$
\begin{aligned}
\mathrm{i} \pi \tau(\mathbf{v})= & \log q_{0}+\mathrm{i} \pi-\log 16+2 \log \left(1-\frac{m^{2}}{\mathbf{v}}\right)+q_{0}\left(\frac{1}{2}-\frac{m^{2}}{\mathbf{v}}+\frac{5 m^{4}}{2 \mathbf{v}^{2}}\right) \\
& +q_{0}^{2}\left(\frac{13}{64}-\frac{13 m^{2}}{16 \mathbf{v}}+\frac{135 m^{4}}{32 \mathbf{v}^{2}}-\frac{109 m^{6}}{16 \mathbf{v}^{3}}+\frac{269 m^{8}}{64 \mathbf{v}^{4}}\right)+\mathscr{O}\left(q_{0}^{3}\right)
\end{aligned}
$$

For simplicity, and also for later convenience, we have introduced a notation that explicitly exhibits only the dependence of $\tau$ on the gauge invariant parameter $\mathbf{v}$.

When the flavors are massless, the effective coupling, which we denote by $\tau_{0}$, is related to $q_{0}$ as follows

$$
\mathrm{i} \pi \tau_{0}=\log q_{0}+\mathrm{i} \pi-\log 16+\frac{1}{2} q_{0}+\frac{13}{64} q_{0}^{2}+\frac{23}{192} q_{0}^{3}+\cdots
$$

It is interesting to observe that the inverse relation can be expressed in terms of modular functions. Indeed, inverting (2.9) we obtain

$$
q_{0}=-16\left(\mathrm{e}^{\mathrm{i} \pi \tau_{0}}+8 \mathrm{e}^{2 \mathrm{i} \pi \tau_{0}}+44 \mathrm{e}^{3 \mathrm{i} \pi \tau_{0}}+\cdots\right)=-\frac{\theta_{2}^{4}\left(\tau_{0}\right)}{\theta_{4}^{4}\left(\tau_{0}\right)}
$$

where the $\theta$ 's are the Jacobi $\theta$-functions ${ }^{1}$. Notice that even in this simple case, $t_{0}$ and $\tau_{0}$ are different and represent two different choices of effective couplings for the massless theory (see also [27]).

\footnotetext{
${ }^{1}$ Notice that this same relation can also be written as $q_{0}=-16 \frac{\eta^{8}\left(4 \tau_{0}\right)}{\eta^{8}\left(\tau_{0}\right)}$ in terms of the Dedekind $\eta$-functions. This expression is amenable of interesting generalizations for superconformal field theories with gauge groups $\mathrm{SU}(N)$ with $N>2$ [22]. We also observe that our coupling is related by a T-duality transformation, $\tau_{0} \rightarrow \tau_{0}+1$, to the one usually considered in the literature $[25,26,23]$ for which the relation $(2.10)$ takes the form $q_{0}=\frac{\theta_{2}^{4}\left(\tau_{0}\right)}{\theta_{3}^{4}\left(\tau_{0}\right)}$.
} 
Let us now consider the same modular function appearing in (2.10), but instead of the massless coupling $\tau_{0}$ let us use as argument the massive coupling $\tau(\mathbf{v})$ :

$$
-\frac{\theta_{2}^{4}(\tau(\mathbf{v}))}{\theta_{4}^{4}(\tau(\mathbf{v}))} \equiv q(\mathbf{v})=-16\left(\mathrm{e}^{\mathrm{i} \pi \tau(\mathbf{v})}+8 \mathrm{e}^{2 \mathrm{i} \pi \tau(\mathbf{v})}+44 \mathrm{e}^{3 \mathrm{i} \pi \tau(\mathbf{v})}+\cdots\right) .
$$

Taking the logarithm of $q(\mathbf{v})$, we then get

$$
\begin{aligned}
\mathrm{i} \pi t(\mathbf{v}) & \equiv \log q(\mathbf{v}) \\
& =\log q_{0}+2 \log \left(1-\frac{m^{2}}{\mathbf{v}}\right)+q_{0} \frac{2 m^{4}}{\mathbf{v}^{2}}+q_{0}^{2}\left(\frac{2 m^{4}}{\mathbf{v}^{2}}-\frac{4 m^{6}}{\mathbf{v}^{3}}+\frac{3 m^{8}}{\mathbf{v}^{4}}\right)+\mathscr{O}\left(q_{0}^{3}\right) .
\end{aligned}
$$

This expression has a very nice interpretation. Indeed, let us consider the SW curve for the SU(2) $N_{f}=4$ SYM which, when the flavor masses are as in (2.2), can be written as [28, 22]

$$
y^{2}=P^{2}(z)-g^{2} Q(z)
$$

where

$$
P(z)=z^{2}-u, \quad Q(z)=\left(z^{2}-m^{2}\right)^{2},
$$

with

$$
u=\frac{1-q_{0}}{1+q_{0}} \mathbf{v}+\frac{2 q_{0}}{1+q_{0}} m^{2}, \quad g^{2}=\frac{4 q_{0}}{\left(1+q_{0}\right)^{2}} .
$$

The curve (2.13) describes a torus whose complex structure parameter is

$$
\tau=\frac{\partial a_{D}}{\partial a}
$$

where $a$ and $a_{D}$ are the periods of the SW 1-form differential $\lambda$

$$
a=\frac{1}{2 \mathrm{i} \pi} \oint_{\gamma} \lambda, \quad a_{D}=\frac{1}{2 \mathrm{i} \pi} \oint_{\widetilde{\gamma}} \lambda
$$

computed around a basis of dual cycles $(\gamma, \widetilde{\gamma})$ normalized in such a way that $\gamma \circ \widetilde{\gamma}=1$. The SW differential $\lambda$ can be written as

$$
\lambda=z \Psi^{\prime}(z) d z
$$

with

$$
\Psi(z)=\log \frac{P(z)+\sqrt{P^{2}(z)-g^{2} Q(z)}}{\mu^{2}} .
$$

Using this information, one can compute $\tau$ and check that, when it is expanded in powers of $q_{0}$, it coincides precisely with the effective gauge coupling (2.8). Other interesting quantities that characterize the curve (2.13) are the anharmonic ratios of the four roots of the equation $y^{2}=0$. It is quite easy to see that these roots are

$$
\begin{aligned}
& e_{1}=+\sqrt{\frac{u-g m^{2}}{1-g}}, \quad e_{2}=-\sqrt{\frac{u+g m^{2}}{1+g}}, \\
& e_{3}=-\sqrt{\frac{u-g m^{2}}{1-g}}, \quad e_{4}=+\sqrt{\frac{u+g m^{2}}{1+g}},
\end{aligned}
$$


and that a corresponding anharmonic ratio is

$$
\zeta \equiv \frac{\left(e_{3}-e_{2}\right)\left(e_{1}-e_{4}\right)}{\left(e_{1}-e_{2}\right)\left(e_{3}-e_{4}\right)}=\frac{\mathbf{v}-\frac{2 q_{0}}{1+q_{0}} m^{2}-\sqrt{\left(\mathbf{v}-\frac{2 q_{0}}{1+q_{0}} m^{2}\right)^{2}-\frac{4 q_{0}}{\left(1+q_{0}\right)^{2}}\left(\mathbf{v}-m^{2}\right)^{2}}}{\mathbf{v}-\frac{2 q_{0}}{1+q_{0}} m^{2}+\sqrt{\left(\mathbf{v}-\frac{2 q_{0}}{1+q_{0}} m^{2}\right)^{2}-\frac{4 q_{0}}{\left(1+q_{0}\right)^{2}}\left(\mathbf{v}-m^{2}\right)^{2}}} .
$$

It is easy to see that the expansion of $\log \zeta$ in powers of $q_{0}$ perfectly matches the expression found in (2.12) and thus we are led to the identification

$$
\mathrm{i} \pi t(\mathbf{v})=\log \zeta .
$$

This is not surprising since the relation between the anharmonic ratio $\zeta$ and the complex structure parameter $\tau$ of a curve like (2.13) is precisely $\log \zeta=-\frac{\theta_{2}^{4}(\tau)}{\theta_{4}^{4}(\tau)}$, namely the same relation between $t(\mathbf{v})$ and $\tau(\mathbf{v})$ implied by (2.11) and (2.12).

We observe observe that the right hand side of (2.21) can be nicely written in terms of the polynomials $P$ and $Q$ of the SW curve describing the theory at the so-called "enhançon vacuum" [22]. This is the specific point of the quantum moduli space corresponding to $\mathbf{v}=0$ which describes a classical extended brane configuration resembling that of the enhançon ring [29]. In the enhançon vacuum we therefore have $u=\frac{2 q_{0}}{1+q_{0}} m^{2}$, and the polynomials $P$ and $Q$ become

$$
\widetilde{P}(z)=z^{2}-\frac{2 q_{0}}{1+q_{0}} m^{2} \quad \widetilde{Q}(z)=\left(z^{2}-m^{2}\right)^{2} .
$$

Then, from (2.21) and (2.22) it is easy to realize that

$$
\mathrm{i} \pi t(\mathbf{v})=\left.\log \frac{\widetilde{P}(z)-\sqrt{\widetilde{P}^{2}(z)-g^{2} \widetilde{Q}(z)}}{\widetilde{P}(z)+\sqrt{\widetilde{P}^{2}(z)-g^{2} \widetilde{Q}(z)}}\right|_{z^{2}=\mathbf{v}} .
$$

Using the information encoded in the SW curve it is also possible to compute the exact quantum correlators $\left\langle\operatorname{tr} \phi_{0}^{\ell}\right\rangle$ forming the chiral ring elements of the gauge theory. These correlators are in fact given by the integral

$$
\left\langle\operatorname{tr} \phi_{0}^{\ell}\right\rangle=\oint_{\gamma} \frac{d w}{2 \mathrm{i} \pi} w^{\ell} \Psi^{\prime}(w) .
$$

or alternatively, they can be obtained by expanding the generating functional [21]

$$
\left\langle\operatorname{tr} \frac{1}{z-\phi_{0}}\right\rangle=\Psi^{\prime}(z) \text {. }
$$

Integrating (2.26) with respect to $z$, it is easy to find

$$
\left\langle\operatorname{tr} \log \frac{z-\phi_{0}}{\mu}\right\rangle=\log \frac{P(z)+\sqrt{P(z)^{2}-g^{2} Q(z)}}{\mu^{2}}-\log \left(1+\sqrt{1-g^{2}}\right),
$$

where the integration constant has been fixed in order to match the $\mathscr{O}\left(z^{0}\right)$ terms in the expansion for large $z$ in both sides. With some further straightforward algebra, we can rewrite the right hand side of (2.27) in the following form

$$
\left\langle\operatorname{tr} \log \frac{z-\phi_{0}}{\mu}\right\rangle=\frac{1}{2} \log \frac{P(z)+\sqrt{P(z)^{2}-g^{2} Q(z)}}{P(z)-\sqrt{P(z)^{2}-g^{2} Q(z)}}+\frac{1}{2} \log \frac{Q(z)}{\mu^{4}}+\frac{1}{2} \log q_{0} .
$$

This expression will be essential in the next section to write the exact (i.e. all order in the instanton expansion) gravitational profile of the twisted scalar field $t$ emitted by the system of fractional D3 branes, and to relate it with the dual gauge theory coupling. 


\section{The exact $t$ profile emitted by fractional D3-branes and D-instantons}

The fractional D3-branes in the $\mathbb{Z}_{2}$ orbifold are gravitational sources for a non-trivial metric and a 4-form R-R potential from the untwisted sectors, and for two scalars, $b$ and $c$, from the twisted NS-NS and R-R sectors respectively (see for instance [3]). While the emitted untwisted fields can propagate in all six directions transverse to the D3-branes, the twisted scalars only propagate in the complex plane transverse to the D3-brane world-volume which is not affected by the orbifold projection and which we parametrize with a complex coordinate $\mathbf{x}$. A system of fractional D3branes distributed on this plane therefore generate a non-trivial dependence of the fields $b$ and $c$ on x. The twisted scalars are conveniently combined in a complex field

$$
t=c+\tau b
$$

where here $\tau$ stands for the axio-dilaton of the type IIB string theory. For simplicity we assume that the axion is trivial and that there are no branes other than the fractional D3 branes so that the dilaton does not run. Thus, in this case we simply have $\tau=\mathrm{i} / g_{s}$ where $g_{s}$ is the string coupling constant. The field $t$ is actually part of a chiral bulk superfield $T$ whose structure is schematically given by

$$
T=t+\cdots+\theta^{4} \frac{\partial^{2}}{\partial \mathbf{x}^{2}} \bar{t}+\cdots
$$

with dots denoting the supersymmetric descendants of $t$ and $\bar{t}$ being the complex conjugate of $t$.

The profile of the twisted scalar $t$ emitted by a system of fractional D3-branes can be derived by solving the classical field equations that follow from the bulk action containing the kinetic terms and the source action describing the emission from the fractional D3-branes. At the perturbative level this profile was obtained long ago in Ref.s [3] -[7] and for a system of $N_{0}$ branes of type 0 and $N_{1}$ branes of type 1 located at the origin is

$$
\mathrm{i} \pi t=\mathrm{i} \pi t_{0}-2\left(N_{0}-N_{1}\right) \log \frac{\mathbf{x}}{\mathbf{x}_{0}}
$$

where $t_{0}=\mathrm{i} /\left(2 g_{s}\right)$ and $\mathbf{x}_{0}$ is an arbitrary length scale. It is convenient to introduce the quantities

$$
z=\frac{\mathbf{x}}{2 \pi \alpha^{\prime}} \quad \text { and } \quad \mu=\frac{\mathbf{x}_{0}}{2 \pi \alpha^{\prime}}
$$

with mass dimension 1, and rewrite the solution (3.3) as follows

$$
\mathrm{i} \pi t=\mathrm{i} \pi t_{0}-2\left(N_{0}-N_{1}\right) \log \frac{z}{\mu} .
$$

Note that in the conformal cases $\left(N_{0}=N_{1}\right)$, we simply have

$$
t=t_{0} .
$$

Let us now consider a more general configuration in which the D3-branes are not all at the origin. This amounts to giving the adjoint scalars non-vanishing vacuum expectation values as in (2.1) (from now on we focus again only on the case $N_{0}=N_{1}=2$ ). Then, one can show that the $t$ profile corresponding to such a configuration is

$$
\mathrm{i} \pi t=\mathrm{i} \pi t_{0}-2 \operatorname{tr} \log \frac{z-\left\langle\phi_{0}\right\rangle}{\mu}+2 \operatorname{tr} \log \frac{z-\left\langle\phi_{1}\right\rangle}{\mu}=\mathrm{i} \pi t_{0}+2 \log \frac{z^{2}-m^{2}}{z^{2}-a^{2}} .
$$


It is not difficult to realize that this $t$ field satisfies the following differential equation

$$
\square t=8 J_{\mathrm{cl}} \delta^{2}(z)
$$

with

$$
J_{\mathrm{cl}}=\sum_{\ell=1}^{\infty} \frac{\mathrm{i}}{\ell !}\left(\operatorname{tr}\left\langle\phi_{0}\right\rangle^{\ell}-\operatorname{tr}\left\langle\phi_{1}\right\rangle^{\ell}\right) \frac{\partial^{\ell}}{\partial z^{\ell}}=\mathrm{i} \operatorname{tr} \mathrm{e}^{\mathrm{i} \bar{p}\left\langle\phi_{0}\right\rangle}-\mathrm{i} \operatorname{tr} \mathrm{e}^{\mathrm{i} \bar{p}\left\langle\phi_{1}\right\rangle}
$$

where in the second step we introduced the momentum operator conjugate to $z$, that is $\bar{p}=-\mathrm{i} \partial / \partial z$.

The current $J_{\mathrm{cl}}$ has a nice interpretation in terms of disk diagrams describing the couplings among the closed string twisted fields and the massless open string excitations of the fractional D3-branes. Indeed, by considering the interactions of the NS-NS scalar $b$ (whose vertex operator we denote by $V_{b}$ ) with the scalar $\phi_{0}$ (whose vertex we denote by $V_{\phi_{0}}$ ), we find

$$
\sum_{\ell=0}^{\infty} \frac{1}{\ell !}\langle\underbrace{V_{\phi_{0}} \cdots V_{\phi_{0}}}_{\ell} V_{b}\rangle_{\mathrm{D}_{0}}=\frac{\pi}{g_{s}} \sum_{\ell=0}^{\infty} \frac{1}{\ell !} \operatorname{tr}\left\langle\phi_{0}\right\rangle^{\ell}(\mathrm{i} \bar{p})^{\ell} b=\frac{\pi}{g_{s}} \operatorname{tr} \mathrm{e}^{\mathrm{i} \bar{p}\left\langle\phi_{0}\right\rangle} b .
$$

This result follows by computing the correlation functions of the vertex operators using standard CFT techniques as discussed for example in [19] and by frozing the scalars to their vacuum expectation values. A completely similar calculation can be performed with the scalar $\phi_{1}$ of the type 1 branes leading to

$$
-\frac{\pi}{g_{s}} \operatorname{tr}^{\mathrm{i}} \mathrm{e}^{\bar{\rho}\left\langle\phi_{1}\right\rangle} b
$$

where the extra sign comes from the fact that branes of type 1 have opposite $b$-charge with respect of those of type 0 . Adding an analogous term describing the interactions of the R-R twisted scalar $c$, we can write the total contribution to the effective action as

$$
-\mathrm{i} \pi\left(\operatorname{tr} \mathrm{e}^{\mathrm{i} \bar{p}\left\langle\phi_{0}\right\rangle}-\operatorname{tre}^{\mathrm{i} \bar{p}\left\langle\phi_{1}\right\rangle}\right) \bar{t} .
$$

Supersymmetry requires that this interaction must be accompanied by other structures (that could also be computed from string diagrams with extra fermionic insertions) in such a way that the effective action follows from a holomorphic prepotential. As discussed in [19]-[22] such a prepotential is obtained simply by promoting the bulk and boundary scalars to the corresponding chiral superfields. Denoting by $\delta T$ the fluctuation part of $T$, one finds in particular the following term

$$
\delta F_{\mathrm{cl}}=\mathrm{i} \pi\left(\operatorname{tr} \mathrm{e}^{\mathrm{i} \bar{p} \Phi_{0}}-\operatorname{tr}^{\mathrm{i} \bar{p} \Phi_{1}}\right) \frac{\delta T}{\bar{p}^{2}}+\cdots
$$

where the dots represent interactions of higher orders in $\delta T$. The effective action follows upon integrating the prepotential over $d^{4} \theta$; when all four $\theta$ 's are taken from $\delta T$ and the superfields $\Phi_{0}$ and $\Phi_{1}$ are frozen to their vacuum expectation values, we recover precisely the interaction (3.12). The classical current (3.9) therefore is associated to a source term for $t$ and is related to the prepotential (3.13) in the following way

$$
J_{\mathrm{cl}}=\left.\frac{\bar{p}^{2}}{\pi} \frac{\delta F_{\mathrm{cl}}}{\delta T}\right|_{\Phi \rightarrow\langle\Phi\rangle}
$$


Let us now investigate how the classical profile (3.7) changes when non-perturbative effects due to gauge instantons are taken into account. In our brane set-up, instantons are introduced by adding fractional $\mathrm{D}(-1)$-branes. Since we neglect the dynamics on the branes of type 1, we only consider the effects produced by adding $k \mathrm{D}$-instantons of type 0 . The physical excitations of the open strings with at least one end-point on the $\mathrm{D}(-1)$-branes account for the instanton moduli which we collectively denote as $\mathscr{M}_{k}$. They consist of the neutral sector, corresponding to $\mathrm{D}(-1) / \mathrm{D}(-1)$ open strings that do not transform under the gauge group, and of the charged and flavored sectors arising respectively from the $\mathrm{D}(-1) / \mathrm{D} 3_{0}$ and $\mathrm{D}(-1) / \mathrm{D} 3_{1}$ open strings. The complete list of instanton moduli and their transformation properties can be found in [21, 22]. Here we just recall that among the neutral moduli we have the bosonic and fermionic Goldstone modes of the supertranslations of the D3-brane world-volume which are broken by the D-instantons and which are identified with the superspace coordinates $x$ and $\theta$, and a complex scalar $\chi$ transforming in the adjoint representation of the instanton symmetry group $\mathrm{U}(k)$, whose eigenvalues describe the position of the D-instantons in the un-orbifolded directions transverse to the fractional D3-branes.

In order to find the non-perturbative $t$ profile we first compute the instanton induced prepotential $F_{\text {n.p. }}$ from which the non-perturbative source current $J_{\text {n.p. }}$ can be derived following a procedure similar to the one outlined for the classical current $J_{\mathrm{cl}}$. The non-perturbative prepotential is defined as

$$
F_{\text {n.p. }}=\sum_{k} \int d \widehat{\mathscr{M}}_{k} \mathrm{e}^{-S_{\text {inst }}\left(\mathscr{M}_{k}, \Phi, T\right)}
$$

where the integral is performed over the centered moduli $\widehat{\mathscr{M}_{k}}$, which include all moduli except the superspace coordinates $x$ and $\theta$. Here $S_{\text {inst }}\left(\mathscr{M}_{k}, \Phi, T\right)$ is the instanton action, describing the interactions of the instanton moduli with the boundary and bulk superfields. As explained in [21], such an action is

$$
S_{\text {inst }}\left(\mathscr{M}_{k}, \Phi, T\right)=-k \mathrm{i} \pi t_{0}+S_{\text {inst }}^{\prime}\left(\mathscr{M}_{k}, \Phi\right)-\mathrm{i} \pi \operatorname{tr}_{k} \mathrm{e}^{\mathrm{i} \bar{p} \chi} \delta T+\ldots
$$

where $S_{\mathrm{inst}}^{\prime}$ is the part accounting for the interactions of the moduli among themselves and with the fields in the vector multiplet, and $\operatorname{tr}_{k}$ means trace over the $\mathrm{U}(k)$ indices. Inserting (3.16) in (3.15), to linear order in $\delta T$ we find

$$
\delta F_{\text {n.p. }}=\mathrm{i} \pi \delta T \sum_{k} q_{0}^{k} \int d \widehat{\mathscr{M}}_{k} \mathrm{e}^{-S_{\text {inst }}^{\prime}\left(\mathscr{M}_{k}, \Phi\right)} \operatorname{tr}_{k} \mathrm{e}^{\mathrm{i} \bar{p} \chi}
$$

The integration over the moduli space can be explicitly performed using localization techniques and Nekrasov's approach to the multi-instanton calculus $[16,17]$. This amounts to first define the deformed instanton partition function

$$
Z_{\text {inst }}=\sum_{k} q_{0}^{k} \int d \mathscr{M}_{k} \mathrm{e}^{-S_{\text {inst }}^{\prime}\left(\mathscr{M}_{k}, \Phi ; \varepsilon_{1}, \varepsilon_{2}\right)}
$$

where $\varepsilon_{1}$ and $\varepsilon_{2}$ are deformation parameters which in our string set-up can be introduced by putting the brane system in a graviphoton background [30,31], and then to compute the prepotential according to

$$
F_{\text {n.p. }}=-\lim _{\varepsilon_{1}, \varepsilon_{2} \rightarrow 0} \varepsilon_{1} \varepsilon_{2} \log Z_{\text {inst }} .
$$


The integral appearing in (3.17) is related to the instanton part of the chiral ring elements $\left\langle\operatorname{tr} \phi_{0}^{\ell}\right\rangle$ of the gauge theory on the D3-branes, which can be computed as ${ }^{2}$

$$
\frac{1}{\ell !}\left\langle\operatorname{tr} \phi_{0}^{\ell}\right\rangle_{\text {inst }}=-\frac{1}{(\ell-2) !} \lim _{\varepsilon_{1}, \varepsilon_{2} \rightarrow 0} \frac{\varepsilon_{1} \varepsilon_{2}}{Z_{\text {inst }}} \sum_{k} q_{0}^{k} \int d \mathscr{M}_{k} \mathrm{e}^{-S_{\text {inst }}^{\prime}\left(\mathscr{M}_{k}, \Phi ; \varepsilon_{1}, \varepsilon_{2}\right)} \operatorname{tr}_{k} \chi^{\ell-2}
$$

Notice that the integrals in (3.18) and (3.20) are over all moduli including $x$ and $\theta$, and that in the limit $\varepsilon_{i} \rightarrow 0$ the factor $\varepsilon_{1} \varepsilon_{2}$ in (3.20) compensates for the volume $V \sim \frac{1}{\varepsilon_{1} \varepsilon_{2}}$ of the regularized four dimensional superspace. Plugging (3.20) into (3.17) one gets

$$
\delta F_{\text {n.p. }}=\mathrm{i} \pi\left\langle\operatorname{tr} \mathrm{e}^{\mathrm{i} \bar{p} \Phi_{0}}\right\rangle_{\text {inst }} \frac{\delta T}{\bar{p}^{2}}
$$

which is nothing but the instanton completion of (3.13).

Adding the classical and the instanton contributions we obtain the full source current for $t$ :

$$
J=\left.\frac{\bar{p}^{2}}{\pi} \frac{\delta F}{\delta T}\right|_{\Phi \rightarrow\langle\Phi\rangle}=\mathrm{i}\left\langle\operatorname{tr} \mathrm{e}^{\mathrm{i} \bar{p} \phi_{0}}\right\rangle-\operatorname{tr} \mathrm{e}^{\mathrm{i} \bar{p}\left\langle\phi_{1}\right\rangle}
$$

where $\delta F=\delta F_{\mathrm{cl}}+\delta F_{\text {n.p. }}$. The field equation satisfied by $t$ is therefore

$$
\square t=8 J \delta^{2}(z)=8 \sum_{\ell=0}^{\infty} \frac{\mathrm{i}}{\ell !}\left[\left\langle\operatorname{tr} \phi_{0}^{\ell}\right\rangle-\operatorname{tr}\left\langle\phi_{1}\right\rangle^{\ell}\right] \frac{\partial^{\ell}}{\partial z^{\ell}} \delta^{2}(z)
$$

which is solved by

$$
\begin{aligned}
\mathrm{i} \pi t & =\mathrm{i} \pi t_{0}-2\left\langle\operatorname{tr} \log \frac{z-\phi_{0}}{\mu}\right\rangle+2 \operatorname{tr} \log \frac{z-\left\langle\phi_{1}\right\rangle}{\mu} \\
& =\mathrm{i} \pi t_{0}-2\left\langle\operatorname{tr} \log \frac{z-\phi_{0}}{\mu}\right\rangle+2 \log \frac{\left(z^{2}-m^{2}\right)}{\mu^{2}} .
\end{aligned}
$$

This explicit solution shows that all non-trivial information about the $t$ profile is contained in the ring of chiral correlators of the gauge theory defined on the D3-branes. This chiral ring accounts therefore for the full tower of D-instanton corrections to the gravity solution.

The chiral correlators $\left\langle\operatorname{tr} \phi_{0}^{\ell}\right\rangle$ can be computed from (3.20) using Nekrasov's approach to the multi-instanton calculus. Equivalently (and more efficiently), as we have explained in the previous section, they can be obtained from the SW curve describing the SYM theory. In fact, inserting (2.28) in (3.24) and taking into account the explicit definition of $Q$ given in (2.14), we can obtain the exact expression for the twisted scalar field emitted by the brane system, namely

$$
\mathrm{i} \pi t=\log \frac{P(z)-\sqrt{P^{2}(z)-g^{2} Q(z)}}{P(z)+\sqrt{P^{2}(z)-g^{2} Q(z)}} .
$$

Our result generalizes the one derived in [32] for the pure SU(N) SYM theories using supergravity and M-theory considerations, and is also perfectly consistent with the findings of [18] where the $\mathrm{SU}(N)$ SYM theory is realized in type IIA using D4 branes stretched between two NS branes.

We can therefore say that the methods we have developed provide a microscopic derivation of the supergravity profile for $t$ in which a direct relation with the chiral ring elements of the gauge theory on the source branes is clearly established and the non-perturbative effects are explicitly explained in terms of fractional D-instantons.

\footnotetext{
${ }^{2}$ For details on the derivation of this formula we refer to [19, 20].
} 


\section{Conclusions}

We have considered a fractional D3-brane system in a $\mathbb{Z}_{2}$ orbifold supporting an $\mathscr{N}=2 \mathrm{SYM}$ theory with SU(2) gauge group and $N_{f}=4$ flavors. We have considered the scalar field $t$ from the twisted closed string sector emitted by such a configuration, which, at the tree level, plays the rôle of the gauge coupling on the D3-branes. As it is well known, the fractional D3-branes act as sources for $t$, so that $t$ has a logarithmic profile in the complex direction $z$ transverse to the orbifold; this profile matches the perturbative running of the gauge coupling if the transverse space is identified with the Coulomb branch of the gauge theory. We have taken into account the non-perturbative effects corresponding to the inclusion of (fractional) D-instantons and explicitly shown how they modify the source for $t$ and hence its profile. The moduli space integrals that determine the nonperturbative source terms are related to the ones appearing in the computation of the chiral ring operators of the gauge theory. Through this relation, we can then express the profile of the twisted scalar as the quantum expectation value of its perturbative expression, see (3.24). This, in turn, can be written in terms of the SW curve that describes the effective dynamics of the gauge theory on the Coulomb moduli space, see (3.25).

At the non-perturbative level, the gauge/gravity relation is deeply modified with respect to its perturbative standing. The twisted scalar $t$ can no longer be simply identified with the effective gauge coupling. However, if we consider the situation in which the source D3-branes sit at the "enhançon" vacuum, $\mathbf{v}=0$, the scalar $t(z)$ is still directly, albeit non-trivially, related to the effective coupling $\tau(\mathbf{v})$ when $z^{2}$ is identified with the quantum Coulomb space variable $\mathbf{v}$. Indeed, in this case $t(z)$ is given by (2.24); this expression corresponds, according to (2.22), to the logarithm of the anharmonic ratio $\zeta$ which parametrizes the SW torus. The anharmonic ratio is related to the complex structure $\tau$, namely to the effective gauge coupling, through the modular function appearing in (2.11).

In these proceedings we focused on the conformal SU(2) case, but in [22] we showed that a similar pattern occurs for higher rank conformal gauge theories, and also, after decoupling some flavors, for asymptotically free cases: the twisted scalar emitted by the branes at the enhançon vacuum is related in the gauge/gravity correspondence to the low energy effective couplings via non-trivial modular functions which are generalizations of that appearing in (2.11).

\section{Acknowledgments}

We warmly thank our coauthors of [22], Francesco Fucito, Francisco Morales and Daniel Ricci-Pacifici, for the fruitful and pleasant collaboration. This work is partially supported by the MIUR-PRIN contract 2009-KHZKRX and by INFN through the projects MI12 and TV12.

\section{References}

[1] J. M. Maldacena, The Large N limit of superconformal field theories and supergravity, Adv.Theor.Math.Phys. 2 (1998) 231-252, arXiv: hep-th/9711200 [hep-th] .

[2] I. R. Klebanov and N. A. Nekrasov, Gravity duals of fractional branes and logarithmic RG flow, Nucl.Phys. B574 (2000) 263-274, arXiv: hep-th/9911096 [hep-th] . 
[3] M. Bertolini, P. Di Vecchia, M. Frau, I. Pesando, A. Lerda, and R. Marotta, Fractional D-branes and their gauge duals, JHEP 0102 (2001) 014, arXiv : hep-th/ 0011077.

[4] J. Polchinski, N=2 Gauge / gravity duals, Int.J.Mod.Phys. A16 (2001) 707-718, arXiv:hep-th/0011193 [hep-th].

[5] M. Petrini, R. Russo, and A. Zaffaroni, $N=2$ gauge theories and systems with fractional branes, Nucl.Phys. B608 (2001) 145-161, arXiv: hep-th/0104026 [hep-th] .

[6] M. Bertolini, P. Di Vecchia, M. Frau, A. Lerda, and R. Marotta, $N=2$ gauge theories on systems of fractional D3/D7 branes, Nucl. Phys. B621 (2002) 157-178, arXiv: hep-th/ 0107057.

[7] M. Billo, L. Gallot, and A. Liccardo, Classical geometry and gauge duals for fractional branes on ALE orbifolds, Nucl. Phys. B614 (2001) 254-278, arXiv: hep-th/ 0105258.

[8] N. Dorey, T. J. Hollowood, V. V. Khoze, and M. P. Mattis, The calculus of many instantons, Phys. Rept. 371 (2002) 231-459, arXiv: hep-th/ 0206063.

[9] M. Bianchi, S. Kovacs, and G. Rossi, Instantons and Supersymmetry, Lect. Notes Phys. 737 (2008) 303-470, arXiv:hep-th/0703142 [HEP-TH].

[10] E. Witten, Small Instantons in String Theory, Nucl. Phys. B460 (1996) 541-559, arXiv:hep-th/9511030.

[11] M. R. Douglas, Branes within branes, arXiv:hep-th/9512077.

[12] M. B. Green and M. Gutperle, D-instanton induced interactions on a D3-brane, JHEP 0002 (2000) 014, arXiv:hep-th/0002011.

[13] M. Billo, M. Frau, I. Pesando, F. Fucito, A. Lerda, and A. Liccardo, Classical gauge instantons from open strings, JHEP 0302 (2003) 045, arXiv : hep-th/ 0211250.

[14] N. Seiberg and E. Witten, Monopole condensation, and confinement in $N=2$ supersymmetric Yang-Mills theory, Nucl. Phys. B426 (1994) 19-52, arXiv: hep-th/ 9407087.

[15] N. Seiberg and E. Witten, Monopoles, duality and chiral symmetry breaking in N=2 supersymmetric QCD, Nucl. Phys. B431 (1994) 484-550, arXiv: hep-th/ 9408099.

[16] N. Nekrasov, Seiberg-Witten prepotential from instanton counting, Adv. Theor. Math. Phys. 7 (2004) 831-864, arXiv:hep-th/0206161.

[17] N. Nekrasov and A. Okounkov, Seiberg-Witten theory and random partitions, arXiv:hep-th/0306238.

[18] E. Witten, Solutions of four-dimensional field theories via M theory, Nucl.Phys. B500 (1997) 3-42, arXiv:hep-th/9703166 [hep-th].

[19] M. Billo, M. Frau, L. Giacone, and A. Lerda, Holographic non-perturbative corrections to gauge couplings, JHEP 1108 (2011) 007, arXiv:1105.1869 [hep-th] .

[20] F. Fucito, J. Morales, and D. Pacifici, Multi instanton tests of holography, JHEP 1109 (2011) 120, arXiv:1106.3526 [hep-th].

[21] M. Billo, M. Frau, L. Giacone, and A. Lerda, Non-perturbative gauge couplings from holography, Fortsch.Phys. 60 (2012) 907-913, arXiv:1201.4231 [hep-th] .

[22] M. Billo, M. Frau, F. Fucito, L. Giacone, A. Lerda, J. F. Morales, and D. Ricci-Pacifici, Non-perturbative gauge/gravity correspondence in $N=2$ theories, JHEP 1208 (2012) 166, arXiv:1206.3914 [hep-th]. 
[23] M. Billo, M. Frau, L. Gallot, A. Lerda, and I. Pesando, Deformed N=2 theories, generalized recursion relations and S-duality, arXiv:1302.0686 [hep-th], to appear in JHEP.

[24] R. Flume, F. Fucito, J. F. Morales, and R. Poghossian, Matone's relation in the presence of gravitational couplings, JHEP 0404 (2004) 008, arXiv: hep-th/ 0403057.

[25] T. W. Grimm, A. Klemm, M. Marino, and M. Weiss, Direct Integration of the Topological String, JHEP 0708 (2007) 058, arXiv : hep-th/ 0702187 [HEP-TH] .

[26] L. F. Alday, D. Gaiotto, and Y. Tachikawa, Liouville correlation functions from four-dimensional gauge theories, Lett. Math. Phys. 91 (2010) 167-197, arXiv:0906.3219 [hep-th] .

[27] M. Billo, L. Gallot, A. Lerda, and I. Pesando, F-theoretic vs microscopic description of a conformal $N=2$ SYM theory, JHEP 1011 (2010) 041, arXiv: 1008.5240 [hep-th] .

[28] P. C. Argyres and S. Pelland, Comparing instanton contributions with exact results in N=2 supersymmetric scale invariant theories, JHEP 0003 (2000) 014, arXiv: hep-th/9911255 [hep-th].

[29] F. Benini, M. Bertolini, C. Closset, and S. Cremonesi, The N=2 cascade revisited and the enhancon bearings, Phys.Rev. D79 (2009) 066012, arXiv:0811.2207 [hep-th].

[30] M. Billo, M. Frau, F. Fucito, and A. Lerda, Instanton calculus in R-R background and the topological string, JHEP 0611 (2006) 012, arXiv: hep-th/ 0606013.

[31] K. Ito, H. Nakajima, T. Saka, and S. Sasaki, $N=2$ Instanton Effective Action in $\Omega$-background and D3/D(-1)-brane System in R-R Background, JHEP 1011 (2010) 093, arXiv: 1009.1212 [hep-th].

[32] S. Cremonesi, Transmutation of $N=2$ fractional D3 branes into twisted sector fluxes, J.Phys. A42 (2009), arXiv:0904.2277 [hep-th]. 